\title{
Intraoperative optical coherence tomography for assessing human lymph nodes for metastatic cancer
}

Ryan M. Nolan ${ }^{1,8}$, Steven G. Adie ${ }^{1,9}$, Marina Marjanovic ${ }^{1}$, Eric J. Chaney ${ }^{1}$, Fredrick A. South ${ }^{1,2}$, Guillermo L. Monroy ${ }^{1,3}$, Nathan D. Shemonski 1,2,10, Sarah J. Erickson-Bhatt ${ }^{1}$, Ryan L. Shelton ${ }^{1,8}$, Andrew J. Bower ${ }^{1,2}$, Douglas G. Simpson ${ }^{1,4}$, Kimberly A. Cradock, Z. George Liu' ${ }^{5}$, Partha S. Ray ${ }^{5,6}$ and Stephen A. Boppart ${ }^{1,2,3,7^{*}}$

\begin{abstract}
Background: Evaluation of lymph node (LN) status is an important factor for detecting metastasis and thereby staging breast cancer. Currently utilized clinical techniques involve the surgical disruption and resection of lymphatic structure, whether nodes or axillary contents, for histological examination. While reasonably effective at detection of macrometastasis, the majority of the resected lymph nodes are histologically negative. Improvements need to be made to better detect micrometastasis, minimize or eliminate lymphatic disruption complications, and provide immediate and accurate intraoperative feedback for in vivo cancer staging to better guide surgery.

Methods: We evaluated the use of optical coherence tomography (OCT), a high-resolution, real-time, label-free imaging modality for the intraoperative assessment of human LNs for metastatic disease in patients with breast cancer. We assessed the sensitivity and specificity of double-blinded trained readers who analyzed intraoperative OCT LN images for presence of metastatic disease, using co-registered post-operative histopathology as the gold standard.

Results: Our results suggest that intraoperative OCT examination of LNs is an appropriate real-time, label-free, non-destructive alternative to frozen-section analysis, potentially offering faster interpretation and results to empower superior intraoperative decision-making.

Conclusions: Intraoperative OCT has strong potential to supplement current post-operative histopathology with real-time in situ assessment of LNs to preserve both non-cancerous nodes and their lymphatic vessels, and thus reduce the associated risks and complications from surgical disruption of lymphoid structures following biopsy.
\end{abstract}

Keywords: Breast cancer, Lymph node, Metastasis, Optical coherence tomography, Intraoperative

\section{Background}

The status of lymph nodes (LNs), with or without metastatic disease, is an important factor in staging cancer, determining appropriate therapies and offering a more accurate prognosis since the transport of primary cancer cells via the lymphatic system is one of the main pathways of metastasis to distant organs. Currently, for staging breast cancer, lymph node status is predominantly

\footnotetext{
* Correspondence: boppart@illinois.edu

'Beckman Institute for Advanced Science and Technology, University of Illinois at Urbana-Champaign (UIUC), 405 N. Mathews Ave., Urbana, IL 61801, USA

${ }^{2}$ Department of Electrical and Computer Engineering, UIUC, Illinois, USA Full list of author information is available at the end of the article
}

evaluated via sentinel lymph node biopsy (SLNB), which involves the removal and analysis of the first, or sentinel, node(s) along the lymphatic chain of nodes draining the primary tumor $[1,2]$. During breast cancer lumpectomy or mastectomy, SLNs are identified through the accumulation of a radioactive agent (Technetium-99) and/or blue dye (isosulfan or methylene) within the node, frequently resulting in the resection and submission of multiple nodes for subsequent, time-consuming, frozen-section or post-operative histopathological analysis [3-7].

While SLNB has progressively replaced axillary lymph node dissection (ALND) for the initial evaluation of nodal involvement in breast cancer staging, a recent 
meta-analysis of intraoperative frozen-section analysis (47 studies, 13,062 patients total) has reported a mean sensitivity of $73 \%$ and specificity of $100 \%$ [8]. Though this study illustrates that frozen-section has been reasonably successful in detecting macrometastatic disease (metastatic tumor cell foci $>2 \mathrm{~mm}$ ) in SLNs (94\% sensitivity), micrometastatic disease (tumor cell foci of $0.2-2 \mathrm{~mm}$ ) detection has proven to be evasive (40\% sensitivity), and post-operative hematoxylin and eosin $(H \& E)$ staining has shown false negative rates as high as $77 \%$ [5-9]. Even random axillary sampling procedures and ALND limited to level 1 (lymph nodes located in the axilla) can miss metastases in $20-25 \%$ of cases because of sampling limitations and the challenge of detecting cancer at the early stages $[10,11]$. Additionally, approximately $75 \%$ of resected nodes from all types of breast cancer are diagnosed tumor-free in post-operative permanent sections [10]. Combined with the fact that the controversial practice of delayed ALND as a second procedure after SLNB micrometastasis detection has been shown to be "oncologically meaningless in $90 \%$ of cases" [9], it can be seen that there is an unnecessary increase of surgical cost, time and patient risk of complications, such as lymphedema and/or sensory changes due to disruption and/or obstruction of the lymphatic drainage network $[5,12,13]$. Lymphedema is considered the most significant concern due to the lifelong risk following surgery, because it occurs in 13-27 \% of breast cancer surgery patients, and because it could be refractory to treatment [11]. All of these factors have fueled the ongoing debate of whether frozen-section examination in SLNB is beneficial to the patient due to the rates of surgical complications [11-18]. Thus, an intraoperative method for the in vivo assessment of SLN status that could replace frozen-section analysis by providing immediate and accurate feedback for cancer staging would thus reduce surgical complication risks by reducing the number of normal lymph nodes resected.

Current intraoperative cancer imaging or sensing techniques, such as ultrasound imaging or the detection of radioactive probes, are limited by low spatial resolution $[19,20]$. These techniques, as well as $\mathrm{x}$-ray computed tomography $(\mathrm{CT})$, positron emission tomography (PET) and magnetic resonance imaging (MRI), can provide LN size and general morphological information, but lack the resolution to reliably detect the presence of metastatic deposits smaller than $2 \mathrm{~mm}$ in the LN [21-23]. In several studies, near-infrared (NIR) fluorescence imaging has been demonstrated to offer high sensitivity [24-26]. While these NIR fluorescent dye injections can be followed in real-time, the localization of the dye only shows the presence of the SLN, not whether the SLN contains metastatic disease [24-26]. Additionally, aside from ultrasound imaging, use of these screening modalities is largely disruptive to surgical workflow and prolong the procedure in much the same way as frozen-section. Therefore, the need for a superior technique to immediately, non-invasively and accurately assess LNs for the presence of metastatic disease remains.

Optical coherence tomography (OCT) provides labelfree, real-time, high-resolution, microstructural imaging not possible with other imaging modalities, and is therefore better suited for the more precise localization and detection of tumor tissue [27-32]. OCT is the optical analogue of ultrasound imaging, wherein tissue is illuminated with near-infrared light and the backscattered light is collected to construct non-invasive, depth-resolved tissue images. OCT imaging resolution is roughly a factor of $10-100 \times$ higher than clinical ultrasound imaging, however OCT cannot image as deeply. The 1-2 mm imaging penetration depth (depending on the tissue type) and the cellular-level resolution of OCT enables the imaging and identification of normal LN microstructure, such as the capsule, cortex, follicles and germinal centers [30, 33-36]. Infiltration of metastatic disease in the subcapsular region of a $\mathrm{LN}$ and other morphological changes associated with metastatic involvement can be identified as bright, highly scattering regions (of pixels) correlated to irregularly dense, homogeneous focal areas when real-time scanning of LN tissue is performed prior to and/or immediately following surgical resection [29, 30, 33-35]. Therefore, intraoperative OCT has the potential to assess LN architecture through an intact capsule and even, without having to physically resect the LN for analysis, providing valuable and accurate real-time feedback to the surgeon on the presence or absence of metastatic disease and potentially enabling the preservation of reactive but non-metastatic nodes, reducing the risk of lymphadema.

In this study, we evaluated the sensitivity and specificity of intraoperative three-dimensional OCT (3D-OCT) for the assessment of metastatic disease in SLNs resected during breast cancer surgery, when compared to the goldstandard post-operative histopathological assessment.

\section{Methods}

\section{Subject population}

The study was conducted according to Declaration of Helsinki principles at two sites (University of Illinois at Urbana-Champaign and Carle Foundation Hospital) in accordance to protocols approved by the Institutional Review Boards at the participating institutions. All participants provided informed written consent prior to enrollment for both ex vivo imaging of LNs and obtaining de-identified pathology reports. Eligible patients were ages 18 years or older, diagnosed with breast cancer and scheduled for surgical intervention with sentinel/axillary LN biopsy. This study included SLNB 
for ductal carcinoma in situ (DCIS) cases, although the role of SLNB in DCIS cases remains controversial [9]. Patients with known infectious blood borne diseases, such as hepatitis $B$, hepatitis $C$ and human immunodeficiency virus (HIV), were excluded from this study. No subject was excluded based on race, ethnic group or gender. A total of 51 subjects were imaged, but only 42 were used in post-analysis based on OCT image evaluation inclusion criteria. A summary of the patient demographics and the related tissue and tumor types is shown in Table 1.

\section{Intraoperative OCT system}

The portable system used for this study included a commercial spectral domain OCT system (Bioptigen, Inc.,

Table 1 Summary of patient demographics and clinical characteristics

\begin{tabular}{|c|c|c|c|}
\hline Characteristic & Imaged & Used & Percent \\
\hline Total \# of subjects & 51 & 42 & $82 \%$ \\
\hline \multicolumn{4}{|l|}{ Age, years } \\
\hline Mean & 61.9 & 62.6 & \\
\hline Stand. Dev. & 12.3 & 11.9 & \\
\hline Range & $34-84$ & $38-84$ & \\
\hline$\leq 65$ & 29 & 23 & \\
\hline$>65$ & 22 & 19 & \\
\hline \multicolumn{4}{|l|}{ Tumor type } \\
\hline Ductal & 43 & 35 & $81 \%$ \\
\hline DCIS & 34 & 28 & \\
\hline IDC & 36 & 29 & \\
\hline Lobular & 13 & 12 & $92 \%$ \\
\hline LCIS & 10 & 9 & \\
\hline ILC & 10 & 9 & \\
\hline Micropapillary & 5 & 4 & $80 \%$ \\
\hline Multi-type & 9 & 8 & $89 \%$ \\
\hline \multicolumn{4}{|c|}{ Tumor size (greatest dimension) } \\
\hline$<1 \mathrm{~cm}$ & 15 & 14 & $93 \%$ \\
\hline $1-2 \mathrm{~cm}$ & 23 & 18 & $78 \%$ \\
\hline$>2 \mathrm{~cm}$ & 13 & 10 & $77 \%$ \\
\hline \multicolumn{4}{|l|}{ Tissue imaged } \\
\hline Lymph nodes & 128 & 76 & $59 \%$ \\
\hline Imaging sites & 184 & 99 & $54 \%$ \\
\hline Histopathology + nodes & 118 & 17 & $14 \%$ \\
\hline \multicolumn{4}{|l|}{ Lymph node metastasis size } \\
\hline \multicolumn{4}{|l|}{ (greatest dimension) } \\
\hline$\leq 2 \mathrm{~mm}$ & 1 & 0 & $0 \%$ \\
\hline$>2 \mathrm{~mm}$ & 19 & 17 & $89 \%$ \\
\hline
\end{tabular}

DCIS ductal carcinoma in situ, IDC invasive ductal carcinoma, LCIS lobular carcinoma in situ, ILC invasive lobular carcinoma
633 Davis Dr., Suite 480, Morrisville, NC 27560) that employed a superluminescent diode as an optical source with a center wavelength of $1310 \mathrm{~nm}$. Signal from this source was passed through a 50:50 optical fiber coupler that was equally split between the reference and sample arms, shown in Fig. 1. In the sample arm, galvanometers and objective lenses (with a fixed focus) were used to volumetrically scan the tissue specimen $(5 \times 5 \times 2 \mathrm{~mm})$ at 7.4 frames $/ \mathrm{sec}(6 \mathrm{kHz} \mathrm{A}$-scan rate) via raster collection of $\mathrm{B}$-scans along the $\mathrm{x}-\mathrm{y}$ plane. The acquired image datasets have $11 \mu \mathrm{m}$ axial resolution and $11 \mu \mathrm{m}$ transverse resolution. The power at the tissue specimen was $1 \mathrm{~mW}$, lower than that found in most commercially available laser pointers. Optical signal reflections collected from the sample and reference arms were recombined at the 50:50 optical fiber coupler, collimated, acquired by the spectral line camera, and stored on the system computer. All images were acquired and post-operatively reviewed with a commercial software package (InVivoVue, Bioptigen, Inc.).

\section{Intraoperative data acquisition}

The subjects, as per standard of care, were injected with a radioactive agent (Technetium-99) and/or methylene blue dye prior to the lumpectomy or mastectomy procedure. Axillary LNs were identified by the accumulation of the injected agent and/or dye, resected individually or as an axillary contents specimen, and provided to the intraoperative research staff for 3D-OCT imaging prior to sending the tissue specimen(s) for routine histopathological examination. In a separate study, 3D-OCT imaging of ex vivo rat LNs containing these localization agents, compared to control LNs, demonstrated that the localization agents do not have a significant effect on the optical scattering or absorption properties in OCT images (data not included).

The tissue specimens were placed in sterile Petri dishes on a mounted, micrometer-positioning stage for optimizing the tissue alignment under the OCT sample arm beam to position the surface of the tissue as close to the top of the OCT imaging frame without risking image wrapping. 3D-OCT datasets were recorded from one or more locations per $\mathrm{LN}$, depending on the size of the node, and marked with surgical ink, which was then set with acetic acid for subsequent correlation to histopathology. All tissue specimens were then returned to the operating room staff after no more than $10 \mathrm{~min}$ of OCT imaging (conducted in parallel with the ongoing surgical procedure) and sent for standard histological processing and pathological analysis. This was done under such time constraints so as to minimize impact on surgical procedure work flow, however, this did restrict the number of LNs that could be imaged during each surgery and any additional LNs resected could not be imaged and included in analysis. 


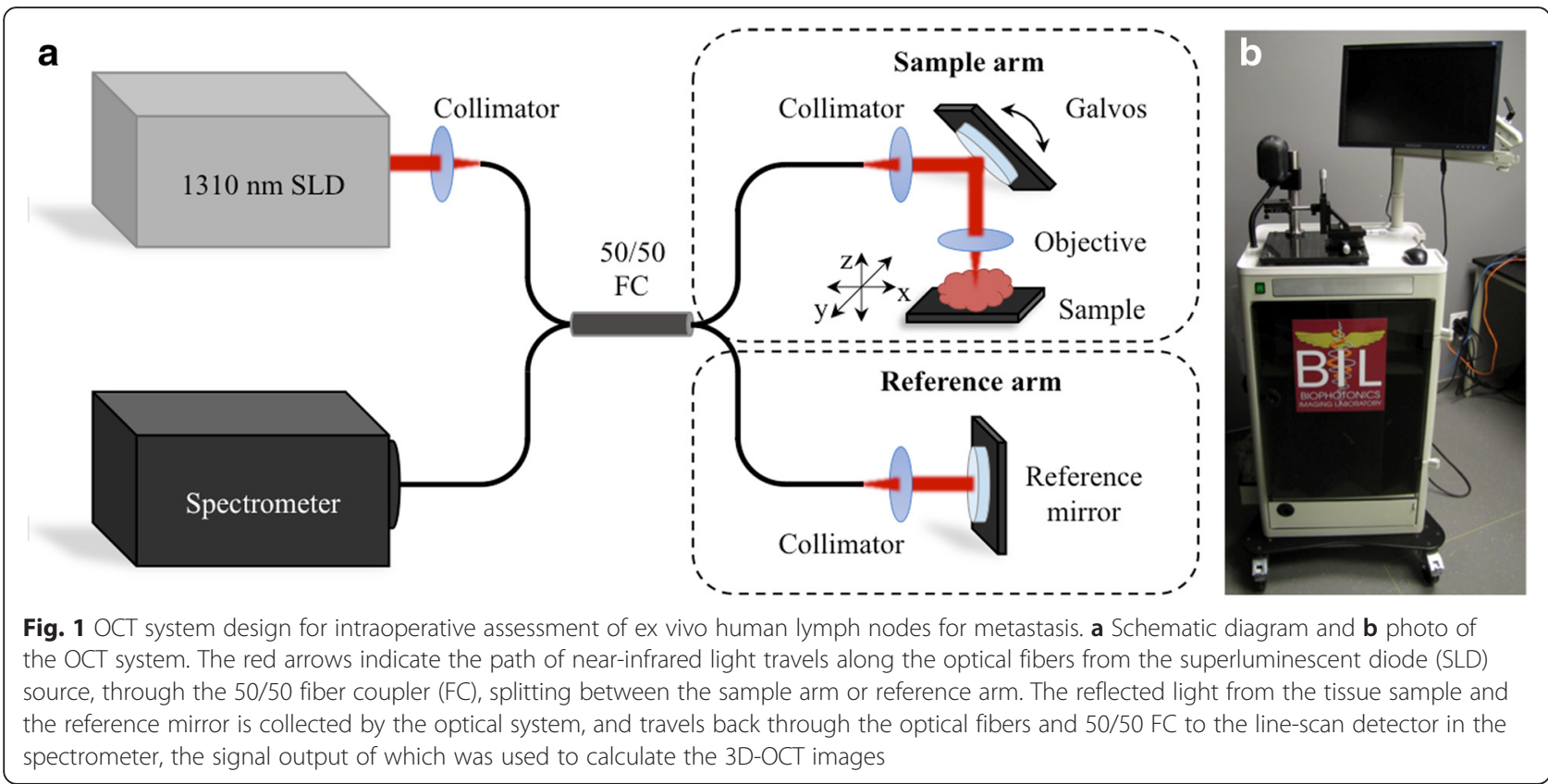

\section{Data and statistical analysis}

We performed a double-blinded study comparing the assessment of OCT datasets to co-registered standard histopathology specimen analysis. A board-certified pathologist examined the co-registered regions on the histology slides to determine the presence of metastatic disease or other significant structural features that may be represented in the OCT image(s). Subsets of the 3D-OCT datasets that correlated with the corresponding histopathology were independently analyzed by three trained OCT readers and classified as "metastatic" or "non-metastatic". These readers were trained using a correlated OCT-histology training set of four sample cases illustrating key OCT features of "metastatic" and "nonmetastatic" cases prior to beginning the blinded analysis. Trained reader analysis of the OCT datasets was guided by the proposed decision tree, shown in Fig. 2, which was developed without input from the three readers. Datasets were not included for reader analysis if LN structure was not imaged due to limitations of OCT penetration depth and any overlying tissue obstruction, such as exceedingly thick surrounding adipose tissue $(>2 \mathrm{~mm})$ overlying the external capsule of the LN. The classification of individual OCT datasets was subsequently consolidated for each OCT observer. Use of a majority voting system for classifying each OCT dataset as cancerous or non-cancerous was supported by receiver operating characteristic (ROC) curve analysis (Fig. 3). Furthermore, LNs that had multiple OCT imaging locations were identified as cancerous if any of the corresponding OCT datasets were classified as cancerous. These OCT analyses for each node were then compared to histological findings, as the gold standard, to calculate system sensitivity and specificity (95\% exact, small sample confidence intervals).

\section{Results}

\section{Intraoperative OCT imaging of human lymph nodes}

Our mobile cart-based intraoperative OCT system was utilized in 51 operations for patients with a preoperative diagnosis of carcinoma, wherein 184 3D-OCT image sets from 128 LNs were acquired immediately following surgical resection and prior to submission of the specimens for standard histopathological processing and analysis (Table 1). The ex vivo imaging sessions required between 5 and $10 \mathrm{~min}$, depending on the size and number of tissue specimens, as well as the number of images acquired, comparatively a fraction of the time required for frozen-section processing and analysis. While most operations had fewer than 5 LNs resected and every LN was able to be imaged within the time constraint, there were several cases where 15-53 LNs were resected. During these cases, only a fraction of the LNs could be imaged and included in the study, without disrupting the surgical procedure workflow. Only 99 3D-OCT images of 76 lymph nodes from 42 operations were used in post-analysis, which, along with surgical imaging time constraints, was based on OCT image evaluation inclusion criteria, namely if the lymph node tissue was present in the correlated histology and OCT image region(s). In the other cases, $\mathrm{LN}$ tissue was absent from the OCT images due to excess overlying adipose tissue. Representative correlated cases of a normal non-metastatic human LN and a cancerous metastatic human LN are shown in Fig. 4. In comparison, representative correlated images 


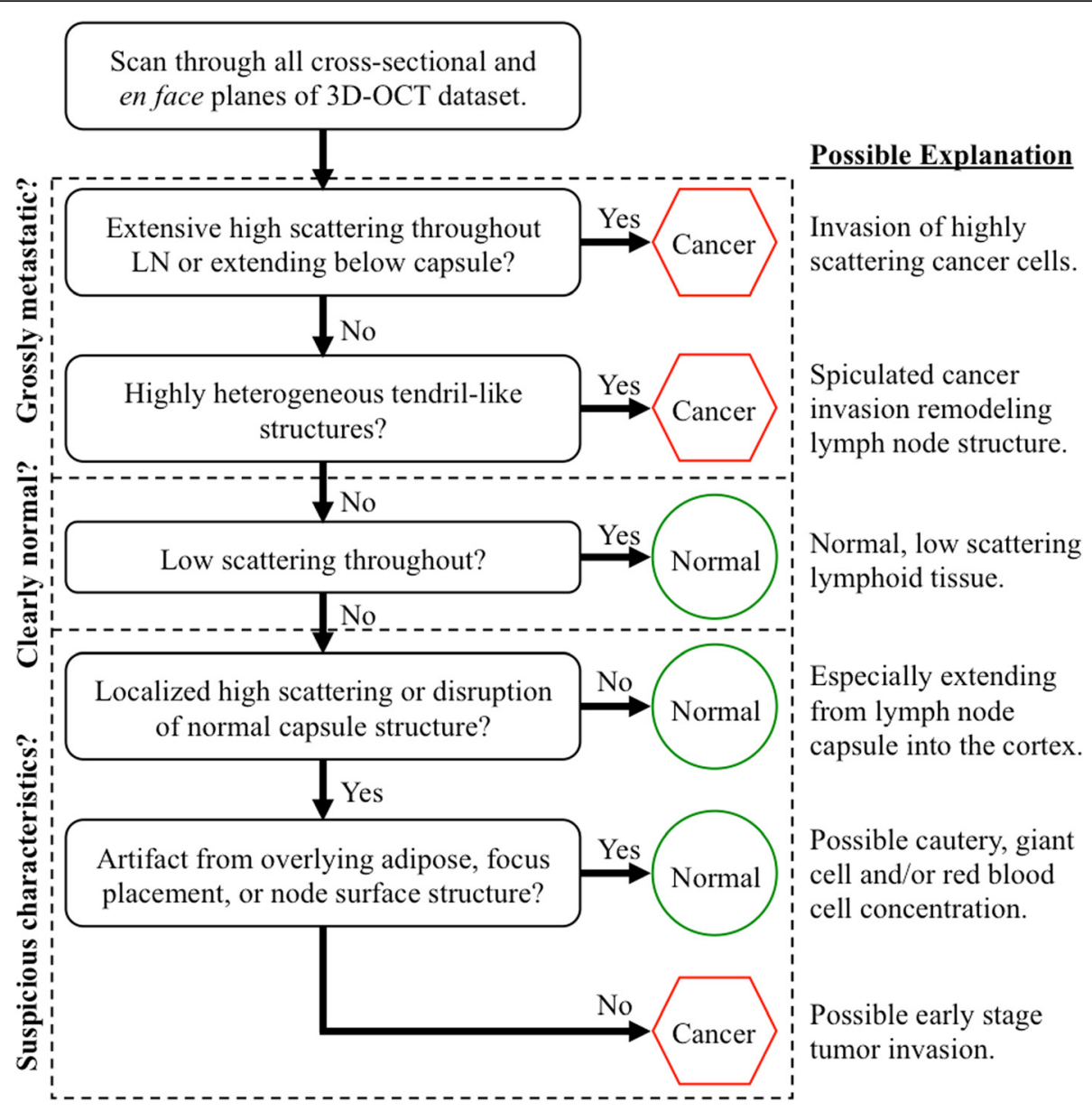

Fig. 2 The decision tree diagram used for analyzing 3D-OCT images. A brief, sample training image set and this decision tree were developed for providing the blinded readers with direction for identifying native and abnormal lymph node anatomical structure, as well as possible image artifacts from imaging limitations in surgery. Each trained reader classified the OCT datasets as either "metastatic" or "non-metastatic"

of a false positive and a false negative case are shown in Fig. 5.

\section{Decision tree-guided lymph node OCT assessment}

Similar to image-based representations in $\mathrm{x}$-ray mammography, bright white regions in the OCT images indicate dense, highly scattering tissue potentially indicative of metastatic disease. Accordingly, a brief, sample training image set and decision tree (Fig. 2) were developed, from knowledge attained from prior work [30, 33-35]. This provided the three OCT-experienced readers with direction for identifying native LN anatomical structure, irregular highly scattering tissue localization and extension, and possible image artifacts from imaging limitations in surgery, such as too much overlying adipose or dense tissue affecting imaging penetration. Ultimately, each trained reader classified the LN OCT datasets as "metastatic" or "non-metastatic". A majority voting system (2 "metastatic" classifications) was initially anticipated to be the most effective assessment method.
An ROC curve, a standard graphical means for analyzing a binary classifier system (i.e. metastatic vs. nonmetastatic) [37], was utilized to visually validate which of the following methods was most effective for classifying LNs under the presented trained reader, post-operative OCT analysis: single "metastatic" reading from a trained OCT reader, majority vote or unanimous decision. Each individual reader's sensitivity and specificity are also indicated and there is no statistically significant difference between individual readers when comparing each reader's confidence interval. Majority voting, which is not unlike other decision-making procedures in diagnostics, was verified (Fig. 3) to be the most effective assessment method because it is the point quantifiably illustrated as the furthest from the "random guess" line and closest to the "perfect classification" point.

From majority voting OCT reader analysis, sensitivity and specificity were $58.8 \%(95 \% \mathrm{CI}, 32.9-81.6)$ and 81.4 \% (95 \% CI, 69.1-90.3), respectively. Considered in other terms, hypothetically if OCT imaging were 


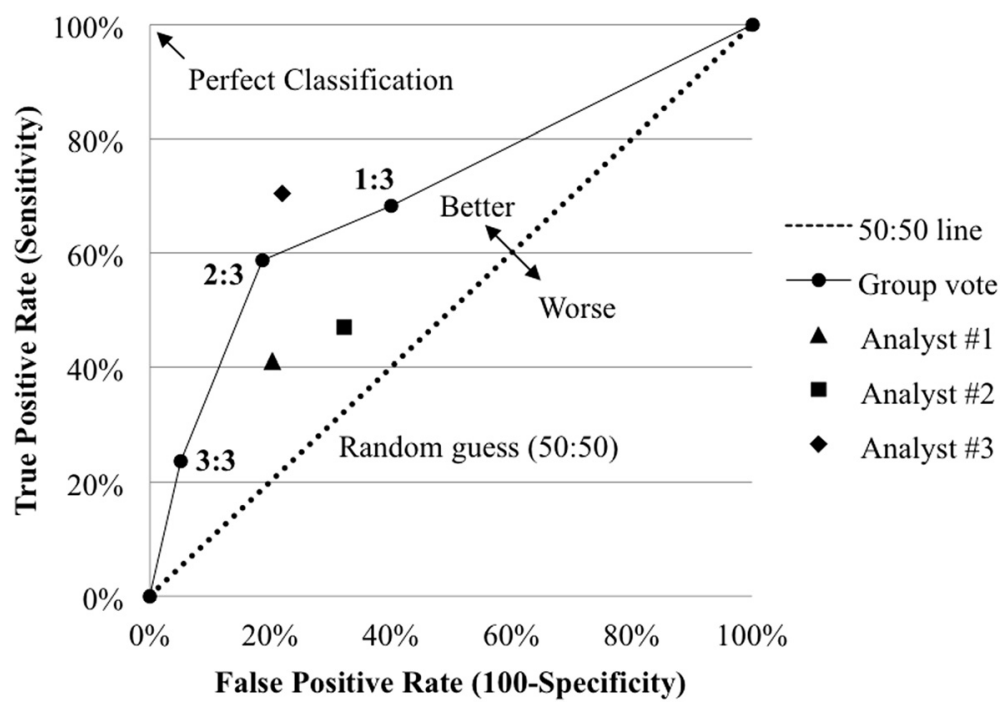

Fig. 3 The receiver operating characteristic (ROC) curve illustrates the comparison of the true and false positive rates as the minimum "positive" vote necessary to label a lymph node metastatic. The three criteria are a single vote (1:3), majority vote (2:3) and unanimous vote (3:3). Majority voting, initially presumed, proved to be the most effective method for trained reader post-operative OCT analysis, since, of the three data points, it is the furthest from the "random guess" (50:50) line and closest to the "perfect classification" limit. Each of the individual OCT reader data are shown for comparison

performed in situ with a hand-held surgical imaging probe, and OCT was used alone to guide LN resection in all of these cases without the use of an injectable tracer or palpation information, the procedures would have resulted in a total of only 21 resected LNs, instead of 76 , over 42 surgeries. This would have reduced the total number of resected LNs by $72 \%$ by preventing the unnecessary resection of 48 non-metastatic LNs, but forgoing the resection of 7 metastatic LNs. In this study, the confidence intervals for sensitivity are wide due to a limited number of positive ("metastatic") sites, while the confidence intervals for specificity are narrower due to a larger number of negative ("non-metastatic") sites imaged. The percentage of resected, positive ("metastatic") LNs in our study was originally in accordance with the national standard (less than $10 \%$ ) $[5,11,13]$. Toward the end of the study, however, we specifically recruited pre-operative biopsy-proven positive cases to increase our proportion of positive LNs to $22 \%$ in an attempt to develop a more balanced statistical analysis.

\section{Discussion}

These results demonstrate that OCT has the potential to be an informative and rapid imaging modality for intraoperative assessment and surgical decision-making of metastatic disease in human lymph nodes. The high specificity indicates a low number of false positives (Fig. 5a and b), which means that when the LN is "non-metastatic", our readers were more readily able to identify normal LN tissue (Fig. 4a and b). This could potentially enable the identification and reduced resection of truly non-reactive, normal LNs and thereby reduce the incidence of lymphedema. However, the moderate sensitivity indicates a poor number of false negatives (Fig. 5c and d), or when the LN is "metastatic", our readers misidentified a cancerous LN for a non-cancerous one. This is in large part due to image artifacts that were also present in many prior ex vivo OCT imaging studies, and though this information was used to develop the brief, sample training image set and decision tree (Fig. 2), reader analysis difficulty persists [30,33-35]. In some of these cases, the OCT signal intensity from a cancerous region(s) was shadowed or reduced by overlying, highly dense stromal tissue, producing a strong OCT signal reflection with diminished signal from deeper tissue (Fig. 5c and d). Additionally, in some cases, thick, overlying adipose tissue restricted the cancerous region in the middle of the LN to the bottom half of the OCT frame, where signal intensity is again weaker from scattering effects, refraction and shadowing, illustrated in Fig. $5 \mathrm{c}$ and d. Furthermore, in several cases, the overlying adipose layer was disrupted and/or lost due to its inherent fragility during histopathological processing. This can make correlation with OCT more difficult since the tissue marking inks can consequently be disrupted and/ or lost during processing, constraining correlations to be made solely through matching LN morphology (Fig. 5d). While real-time, OCT-guided $\mathrm{LN}$ evaluation is nondestructive and less time consuming than frozen-section analysis, a frozen-section preparation provides deeper tissue visualization. Although the imaging team was restricted by our IRB protocol from doing so in this study, 


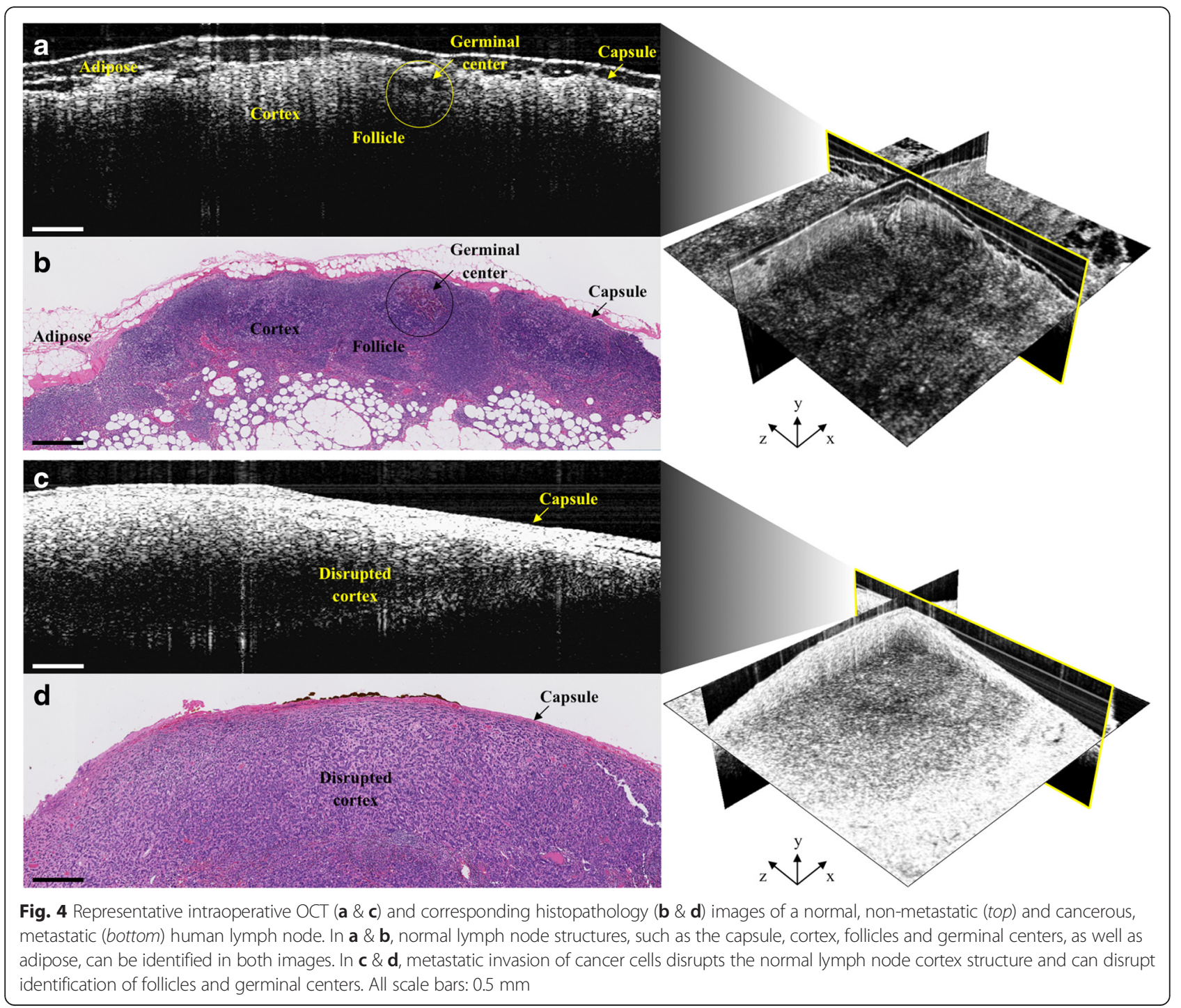

future intraoperative OCT use might require the removal or displacement of any overlying adipose tissue to better expose the LN capsule, maximize OCT imaging depth into the node, and improve overall signal quality through reduced scattering, refraction and shadowing artifacts. For suspicious and/or large tissue samples, hemisection followed by OCT imaging of the corresponding cut surfaces would enable imaging and visualization of deeper LN structures comparable to standard frozensection analysis, while still remaining the faster of the two techniques.

Interestingly, in all false positive 3D-OCT datasets, the corresponding histology revealed crush artifacts and/or grossly reactive, yet histologically non-metastatic, nodules (Fig. 5b), which are much more dense than normal LN tissue and could produce higher optical scattering similar to that from metastatic cell infiltration (Fig. 4c). The crush artifacts could also be attributed to tissue trauma during resection or gross histopathological sectioning, and therefore in situ imaging could reduce the occurrence of these artifacts in the OCT images. Given that the current surgical standard supports resection of suspicious LNs, OCT may offer the most surgical benefit by providing additional means for identifying normal LNs and thereby reduce unnecessary resection of additional LNs beyond the true SLNs, thereby reducing unnecessary surgical disruption of lymphoid tissue and associated lymphatic vessels.

Further improvements in OCT resolution, through the incorporation of computed imaging techniques such as interferometric synthetic aperture microscopy (ISAM) $[38,39]$ and computational adaptive optics (CAO) $[40,41]$ could further enhance the real-time diagnostic capabilities of OCT in the operating room. Use of injectable magnetic nanoparticles [42], such as iron oxide, has shown promise as an alternative to the current radiotracers or dyes, as 


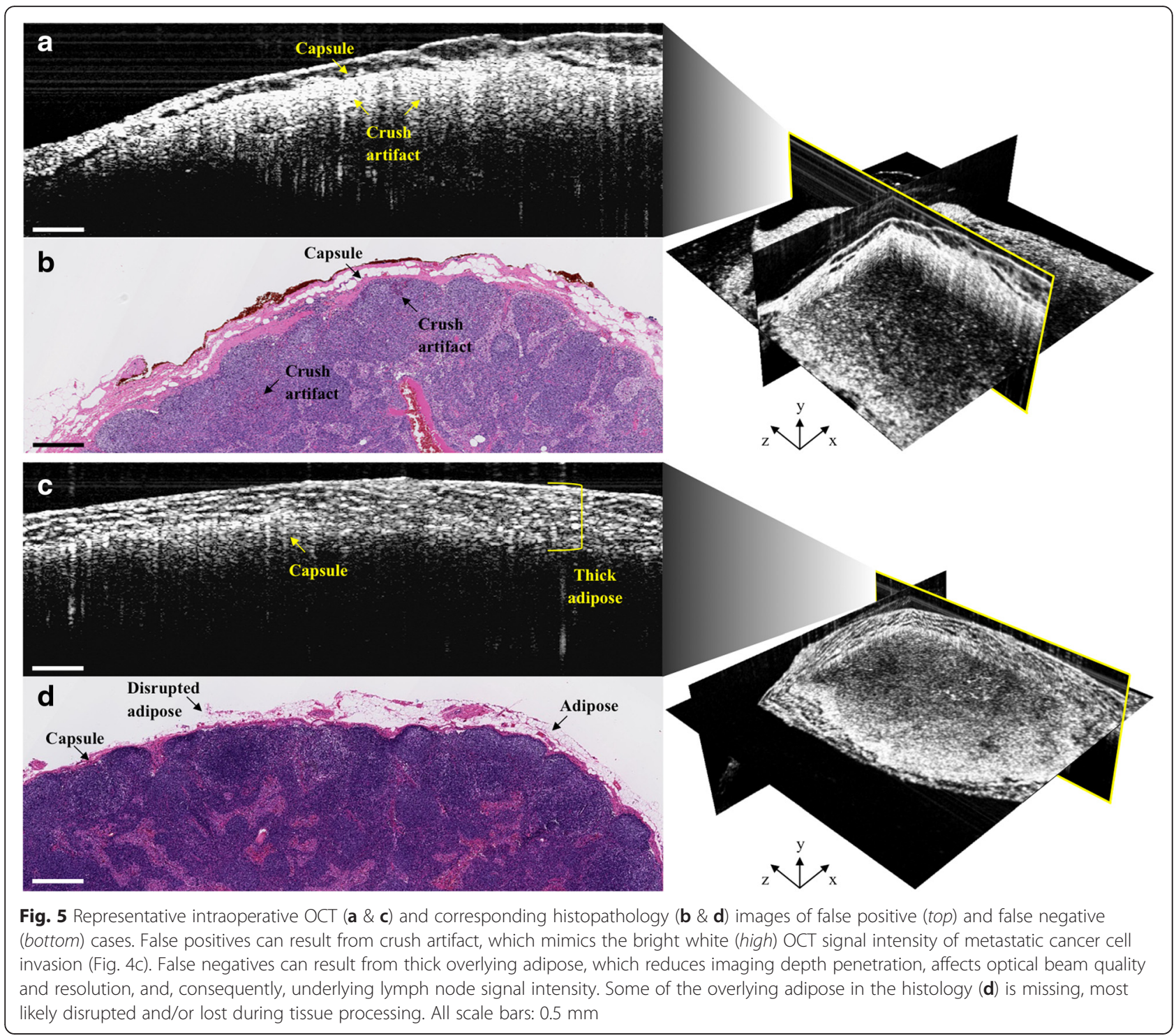

well as a method for improving OCT image quality and cancer detection when coupled with magnetomotive OCT [43]. Optical needle probes used for beam-delivery in OCT systems could be inserted into suspicious in vivo LNs to provide greater OCT imaging depth penetration while minimizing tissue disruption, especially compared to LN hemisection [44-46]. Additionally, improvements in OCT data acquisition rates could facilitate faster intraoperative imaging of all exposed tissue surfaces, whether in vivo or across an ex vivo specimen, to construct a more complete evaluation, especially when coupled with ISAM and CAO [47]. These techniques could be integrated into surgical systems to improve OCT depth resolution and acquisition speed in real-time, and enable further improved detection of metastases not possible with current medical imaging technology. Further improvements and coupling with optical molecular imaging techniques like nonlinear interferometric vibrational imaging (NIVI) of intrinsic molecular markers could produce a more sensitive and accurate color-coded image differentiating cancer from normal tissues [48].

Although all LN metastases evaluated in this study were classified as macrometastases, ongoing work with an intraoperative handheld OCT probe functioning with near-cellular resolution could lead to identification of LN metastases smaller than $2 \mathrm{~mm}$. Additionally, future intraoperative OCT-guided assessment of in situ LNs by the surgeon will incorporate additional key information that will contribute towards diagnosis. Although there are limitations to OCT, such as under-sampling while imaging, and the extended time needed for scanning/ sampling an entire node, these limitations are also present in frozen-section analysis. Furthermore, while our trained readers were blinded during their assessment 
of only the 3D-OCT datasets, intraoperative surgical OCT-guided assessment will be accompanied by far more subject case and procedural knowledge, such as pre-operative findings, LN size, visual and palpable features and the presence of injectable radiotracer and/or dye. Therefore, future studies incorporating intraoperative in vivo 3D-OCT assessment by surgeons will likely facilitate a more practical evaluation of OCT compared to frozen-section analysis. The potential role for a surgical pathologist to review and interpret the real-time 3D-OCT data during the surgical procedure also exists, as an alternative to their role in sectioning, staining and interpreting frozen-section histopathology. While realtime, 3D-OCT transcapsule imaging will not likely replace surgical specimen histopathology (where true cellular and molecular diagnostics are achieved), it does enable advantages for both assessment speed and in vivo imaging, which are preferred over any ex vivo technique when considering tissue conservation. Use of an intraoperative handheld probe for in vivo assessment of breast cancer [49] could also enable LN evaluation prior to removal, and a potential corresponding reduction of risk of complications such as lymphedema. Therefore, OCT could provide a more effective means for $\mathrm{LN}$ assessment and intraoperative cancer staging while potentially reducing unnecessary surgery time and the time and costs associated with extensive frozen-section or postoperative histopathology for the large number of histologically normal LNs.

\section{Conclusion}

Current intraoperative cancer imaging and sensing techniques are limited by low spatial resolution and a clear need remains for a superior technique that can non-invasively and accurately assess LNs for the presence of metastatic disease. Intraoperative OCT has strong potential to supplement current postoperative histopathology with real-time in situ assessment of LNs to preserve both non-cancerous nodes and their lymphatic vessels. Use of such technology could reduce the associated risks and complications from surgical disruption of lymphoid structures following biopsy.

\footnotetext{
Abbreviations

ALND: axillary lymph node dissection; CAO: computational adaptive optics: Cl: confidence interval; CT: x-ray computed tomography; DCIS: ductal carcinoma in situ; FC: fiber coupler; H\&E: hematoxylin and eosin; HIV: human immunodeficiency virus; IDC: invasive ductal carcinoma; ILC: invasive lobular carcinoma; ISAM: interferometric synthetic aperture microscopy; LCIS: Iobular carcinoma in situ; LN: lymph node; MRI: magnetic resonance imaging; NIR: near-infrared; NIVI: nonlinear interferometric vibrational imaging; OCT: optical coherence tomography; PET: positron emission tomography; ROC: receiver operating characteristic; SLD: superluminescent diode; SLNB: sentinel lymph node biopsy; UIUC: University of Illinois at Urbana-Champaign.
}

\section{Competing interests}

Stephen A. Boppart is co-founder, Chief Medical Officer and consultant for Diagnostic Photonics, Inc., which is licensing intellectual property from the University of Illinois at Urbana-Champaign related to Interferometric Synthetic Aperture Microscopy. He also receives royalties for patents licensed by MIT related to optical coherence tomography. For the remaining authors, none were declared.

\section{Authors' contributions}

RMN participated in the intraoperative OCT imaging, histology digitizing and OCT correlation, histology analysis, decision tree design, statistical analysis and drafting the manuscript. SGA participated in the intraoperative OCT imaging, decision tree design, trained reader analysis, statistical analysis and drafting the manuscript. MM participated in the study design, histology analysis, trained reader analysis and drafting the manuscript. EJC participated in the intraoperative OCT imaging, histology analysis and drafting the manuscript. FAS participated in the intraoperative OCT imaging, histology digitizing and OCT correlation, and drafting the manuscript. GLM participated in the intraoperative OCT imaging and trained reader analysis. NDS, SJE and RLS participated in the decision tree design and trained reader analysis. AJB participated in the trained reader analysis. DGS participated in the study design, statistical analysis and drafting the manuscript. KAC performed the surgeries. ZGL conducted the histology analysis. PSR performed the surgeries and participated in the drafting of the manuscript. SAB led the study design and participated in the decision tree design, statistical analysis and drafting of the manuscript. All authors have read and have approved this manuscript for publication.

\section{Acknowledgements}

The authors would like to thank Barbara Hall from the Carle Cancer Center and Carle Foundation Hospital Research Office, as well as all the surgical and nursing staff for providing research and logistical support for this study. We also thank Darold Spillman from the Beckman Institute for Advanced Science and Technology for operations and information technology support. This research was supported in part by grants from the National Institutes of Health (R01 EB012479 and R01 CA166309). Additional information can be found at: http://biophotonics.illinois.edu.

\section{Author details}

'Beckman Institute for Advanced Science and Technology, University of Illinois at Urbana-Champaign (UIUC), 405 N. Mathews Ave., Urbana, IL 61801, USA. ${ }^{2}$ Department of Electrical and Computer Engineering, UIUC, Illinois, USA. ${ }^{3}$ Department of Bioengineering, UIUC, Illinois, USA. ${ }^{4}$ Department of Statistics, UIUC, Illinois, USA. ${ }^{5}$ Carle Foundation Hospital, Urbana, IL, USA. ${ }^{6}$ Department of Surgery, University of Illinois College of Medicine at Urbana-Champaign and Carle Cancer Center, Urbana, IL, USA. ${ }^{7}$ Department of Internal Medicine, UIUC, Illinois, USA. ${ }^{8}$ PhotoniCare, Inc., Champaign, IL, USA. ${ }^{9}$ Department of Biomedical Engineering, Cornell University, Ithaca, NY, USA. ${ }^{10}$ Carl Zeiss Meditec, Inc., Dublin, CA, USA.

Received: 21 July 2015 Accepted: 17 February 2016

Published online: 23 February 2016

\section{References}

1. Zengel B, Yararbas U, Sirinocak A, Ozkok G, Denecli AG, Postaci H, et al. Sentinel lymph node biopsy in breast cancer: review on various methodological approaches. Tumori. 2013;99(2):149-53.

2. Veronesi U, Paganelli G, Viale G, Luini A, Zurrida S, Galimberti V, et al. A randomized comparison of sentinel-node biopsy with routine axillary dissection in breast cancer. N Engl J Med. 2003;349(6):546-53.

3. Krag D, Ashikaga T, Harlow SP, Weaver DL. Development of sentinel node targeting technique in breast cancer patients. Breast J. 1998;4(2):67-74.

4. Blessing WD, Stolier AJ, Teng SC, Bolton JS, Fuhrman GM. A comparison of methylene blue and lymphazurin in breast cancer sentinel node mapping. Am J Surg. 2002;184(4):341-5.

5. Ballehaninna UK, Chamberlain RS. Utility of intraoperative frozen section examination of sentinel lymph nodes in ductal carcinoma in situ of the breast. Clin Breast Cancer. 2013;13(5):350-8.

6. Jensen AJ, Naik AM, Pommier RF, Vetto JT, Troxell ML. Factors influencing accuracy of axillary sentinel lymph node frozen section for breast cancer. Am J Surg. 2010;199(5):629-35. 
7. McLaughlin SA, Ochoa-Frongia LM, Patil SM, Cody 3rd HS, Sclafani LM. Influence of frozen-section analysis of sentinel lymph node and lumpectomy margin status on reoperation rates in patients undergoing breast-conservation therapy. J Am Coll Surg. 2008;206(1):76-82.

8. Liu LC, Lang JE, Lu Y, Roe D, Hwang SE, Ewing CA, et al. Intraoperative frozen section analysis of sentinel lymph nodes in breast cancer patients: meta-analysis and single-institution experience. Cancer. 2011;117(2):250-8.

9. Yamada A, Takabe K. Should we examine sentinel lymph nodes during the operation? Gland Surg. 2012;1(3):161-3.

10. Lyman GH, Giuliano AE, Somerfield MR, Benson 3rd AB, Bodurka DC, Burnstein HJ, et al. American Society of Clinical Oncology guideline recommendations for sentinel lymph node biopsy in early-stage breast cancer. J Clin Oncol. 2005;23(30):7703-20.

11. Vitug AF, Newman LA. Complications in breast surgery. Surg Clin North Am. 2007;87(2):431-51.

12. Bernardi S, Bertozzi S, Londero AP, Angione V, Petri R, Giacomuzzi F. Prevalence and risk factors of intraoperative identification failure of sentinel lymph nodes in patients affected by breast cancer. Nucl Med Commun. 2013;34(7):664-73.

13. Ha KY, Parish D, Hamilton R, Wang JC. Fat necrosis in the breast from methylene blue dye injection. Proc (Baylor Univ MedCent. 2013;26(3):298-9.

14. Boler DE, Cabioglu N, Ince U, Esen G, Uras C. Sentinel lymph node biopsy in pure DCIS: Is it necessary? ISRN Surg. 2012;2012:394095.

15. Intra M, Rotmensz N, Veronesi P, Colleoni M, lodice S, Paganelli G, et al. Sentinel node biopsy is not a standard procedure in ductal carcinoma in situ of the breast: the experience of the European Institute of Oncology on 854 patients in 10 years. Ann Surg. 2008;247(2):315-9.

16. Burstein HJ, Polyak K, Wong JS, Lester SC, Kaelin CM. Ductal carcinoma in situ of the breast. N Engl J Med. 2004;350(14):1430-41.

17. Yen TW, Hunt KK, Ross MI, Mirza NQ, Babiera GV, Meric-Bernstam F, et al. Predictors of invasive breast cancer in patients with an initial diagnosis of ductal carcinoma in situ: a guide to selective use of sentinel lymph node biopsy in management of ductal carcinoma in situ. J Am Coll Surg. 2005:200(4):516-26.

18. Cox CE, Nguyen K, Gray RJ, Salud C, Ku NN, Dupont E, et al. Importance of lymphatic mapping in ductal carcinoma in situ (DCIS): why map DCIS? Am Surg. 2001;67(6):513-9.

19. Karwowski JK, Jeffrey RB, McDougall IR, Weigel RJ. Intraoperative ultrasonography improves identification of recurrent thyroid cancer. Surgery. 2002;132(6):924-8.

20. Strong VE, Humm J, Russo P, Jungbluth A, Wong WD, Daghighian F, et al. A novel method to localize antibody-targeted cancer deposits intraoperatively using handheld PET beta and gamma probes. Surg Endosc. 2008;22(2):386-91.

21. Torabi M, Aquino SL, Harisinghani MG. Current concepts in lymph node imaging. J Nucl Med. 2004;45(9):1509-18.

22. Gadd M. Sentinel lymph node biopsy for staging early breast cancer: minimizing the trade-off by maximizing the accuracy. Ann Oncol. 2009:20(6):973-5.

23. Quan ML, McCready D. The evolution of lymph node assessment in breast cancer. J Surg Oncol. 2009;99(4):194-8.

24. Ashitate $\mathrm{Y}$, Hyun $\mathrm{H}$, Kim SH, Lee $\mathrm{JH}$, Henary M, Frangioni JV, et al. Simultaneous mapping of pan and sentinel lymph nodes for real-time image-guided surgery. Theranostics. 2014;4(7):693-700.

25. Meric-Bernstam F, Rasmussen JC, Krishnamurthy S, Tan IC, Zhu B, Wagner JL, et al. Toward nodal staging of axillary lymph node basins through intradermal administration of fluorescent imaging agents. Biomed Opt Express. 2013;5(1):183-96.

26. Hawrysz DJ, Sevick-Muraca EM. Developments toward diagnostic breast cancer imaging using near-infrared optical measurements and fluorescent contrast agents. Neoplasia. 2000;2(5):388-417.

27. Zysk AM, Nguyen FT, Oldenburg AL, Marks DL, Boppart SA. Optical coherence tomography: a review of clinical development from bench to bedside. J Biomed Opt. 2007:12(5):051403.

28. Vakoc BJ, Fukumura D, Jain RK, Bouma BE. Cancer imaging by optical coherence tomography: Preclinical progress and clinical potential. Nat Rev Cancer. 2012;12(5):363-8.

29. McLaughlin RA, Scolaro L, Robbins P, Hamza S, Saunders C, Sampson DD. Imaging of human lymph nodes using optical coherence tomography: potential for staging cancer. Cancer Res. 2010;70(7):2579-84.
30. Nguyen FT, Zysk AM, Chaney EJ, Adie SG, Kotynek JG, Oliphant UJ, et al. Optical coherence tomography: the intraoperative assessment of lymph nodes in breast cancer. IEEE Eng Med Biol Mag. 2010;29(2):63-70.

31. Nguyen FT, Zysk AM, Chaney EJ, Kotynek JG, Oliphant UJ, Bellafiore FJ, et al. Intraoperative evaluation of breast tumor margins with optical coherence tomography. Cancer Res. 2009;69(22):8790-6.

32. Hariri LP, Mino-Kenudson M, Mark EJ, Suter MJ. In vivo optical coherence tomography: the role of the pathologist. Arch Pathol Lab Med. 2012;136(12):1492-501.

33. John R, Adie SG, Chaney EJ, Marjanovic M, Tangella KV, Boppart SA. Three-dimensional optical coherence tomography for optical biopsy of lymph nodes and assessment of metastatic disease. Ann Surg Oncol. 2013;20(11):3685-93.

34. Boppart SA, Luo W, Marks DL, Singletary KW. Optical coherence tomography: feasibility for basic research and image-guided surgery of breast cancer. Breast Cancer Res Treat. 2004;84(2):85-97.

35. Luo W, Nguyen FT, Zysk AM, Ralston TS, Brockenbrough J, Marks DL, et al. Optical biopsy of lymph node morphology using optical coherence tomography. Technol Cancer Res Treat. 2005;4(5):539-48.

36. Willard-Mack CL. Normal structure, function, and histology of lymph nodes. Toxicol Pathol. 2006;34(5):409-24.

37. Pepe MS. The statistical evaluation of medical tests for classification and prediction. New York: Oxford University Press; 2004.

38. Ralston TS, Marks DL, Carney PS, Boppart SA. Interferometric synthetic aperture microscopy. Nat Phys. 2007;3:129-34.

39. Ahmad A, Shemonski ND, Adie SG, Kim H, Hwu WM, Carney PS, et al. Real-time in vivo computed optical interferometric tomography. Nat Photonics. 2013;7(6):444-8.

40. Adie SG, Shemonski ND, Graf BW, Ahmad A, Carney PS, Boppart SA. Guide-star-based computational adaptive optics for broadband interferometric tomography. Appl Phys Lett. 2012;101(22):221117.

41. Adie SG, Graf BW, Ahmad A, Carney PS, Boppart SA. Computational adaptive optics for broadband optical interferometric tomography of biological tissue. Proc Natl Acad Sci U S A. 2012;109(19):7175-80.

42. Thill M, Kurylcio A, Welter R, van Haasteren V, Grosse B, Berclaz G, et al. The Central-European SentiMag study: sentinel lymph node biopsy with superparamagnetic iron oxide (SPIO) vs. radioisotope. Breast. 2014;23(2):175-9.

43. John R, Rezaeipoor R, Adie SG, Chaney EJ, Oldenburg AL, Marjanovic M, et al. In vivo magnetomotive optical molecular imaging using targeted magnetic nanoprobes. Proc Natl Acad Sci U S A. 2010;107(18):8085-90.

44. Kuo WC, Kim J, Shemonski ND, Chaney EJ, Spillman DR, Boppart SA. Real-time three-dimensional optical coherence tomography image-guided core-needle biopsy system. Biomed Opt Express. 2012;3(6):1149-61.

45. Zysk AM, Nguyen FT, Chaney EJ, Kotynek JG, Oliphant UJ, Bellafiore FJ, et al. Clinical feasibility of microscopically-guided breast needle biopsy using a fiber-optic probe with computer-aided detection. Technol Cancer Res Treat. 2009;8(5):315-21.

46. Curatolo A, McLaughlin RA, Quirk BC, Kirk RW, Bourke AG, Wood BA, et al. Ultrasound-guided optical coherence tomography needle probe for the assessment of breast cancer tumor margins. AJR Am J Roentgenol. 2012;199(4):W520-2.

47. Liu YZ, Shemonski ND, Adie SG, Ahmad A, Bower AJ, Carney PS, et al. Computed optical interferometric tomography for high-speed volumetric cellular imaging. Biomed Opt Express. 2014;5(9):2988-3000.

48. Chowdary PD, Jiang Z, Chaney EJ, Benalcazar WA, Marks DL, Gruebele M, et al. Molecular histopathology by spectrally reconstructed nonlinear interferometric vibrational imaging. Cancer Res. 2010;70(23):9562-9.

49. Erickson-Bhatt SJ, Nolan RM, Shemonski ND, Adie SG, Putney J, Darga D, et al. Real-time imaging of the resection bed using a handheld probe to reduce incidence of microscopic positive margins in cancer surgery. Cancer Res. 2015;75(18):3706-12. 